Pacific Journal of Mathematics

THE GEOMETRY OF THE JAMES-HOPF MAPS 


\section{THE GEOMETRY OF THE JAMES-HOPF MAPS}

\section{NichOLAS J. KUHN}

Snaith's splitting of the suspension spectrum of the space $\Omega^{k} \Sigma^{k} X$, for $X$ path connected, into the wedge of the suspension spectra of spaces denoted $D_{k, q} X$, has been of considerable interest to homotopy theorists in recent years. If $\Sigma^{\infty} X$ denotes the suspension spectrum of a space $X$ then this can be restated as

$$
\Sigma^{\infty} \Omega^{k} \Sigma^{k} X \cong \bigvee_{q \geqq 1} \Sigma^{\infty} D_{k, q} X
$$

Projection onto the $q$ th wedge summand and adjunction yield James-Hopf maps $j_{q}: \Omega^{k} \Sigma^{k} X \rightarrow Q D_{k, q} X$, where $Q Y=\underset{\rightarrow}{\lim } \Omega^{k} \Sigma^{k} Y$.

In this paper $I$ study various compatibility $\overrightarrow{\text { relations }}$ which hold among the $j_{q}$ as $X$ is replaced by $\Sigma^{n} X$. In particular, I show that, for $k>n$, the iterated evaluation map $\varepsilon_{n}: \Sigma^{n} \Omega^{k} \Sigma^{k} X \rightarrow \Omega^{k-n} \Sigma^{k} X$ is naturally compatible with the stable splittings of these two spaces. This is done by exhibiting maps $\delta_{k, n}: \Sigma^{n} D_{k, q} X \rightarrow D_{k-n, q} \Sigma^{n} X$ making the following diagram of suspension spectra homotopy commute:

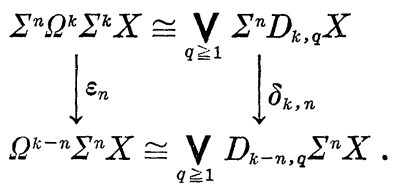

In certain cases, the maps $\delta_{k, n}$ are then identified as standard projection maps. Consequences are then discussed.

Special cases of James-Hopf maps were defined by James [7], Toda [17], and Milgram [13] who fit their maps into the E-H-P sequence and its generalization. Snaith [16] then proved the above mentioned splitting theorem. If $X$ is a Thom space, Kosckorke and Sanderson [9] have given a geometric description of these maps as operations between bordism theories.

J. Caruso, F. Cohen, J. P. May, and L. Taylor $[2,5]$ note that a "Cartan formula" interrelating the maps $j_{q}$ allow them to be defined even for nonconnected spaces. James' original combinatorial construction of the Hopf invariant is used as a model. This version can be used for all of the above applications, and the extension to nonconnected spaces leads to a quite elementary proof of the KahnPriddy theorem $[2,15]$.

Section 1 contains the statements of our compatibility results. In $\S 2$ we apply these results in conjunction with the $\mathrm{E}-\mathrm{H}-\mathrm{P}$ sequence and the Kahn-Priddy theorem. In particular, we prove a "delooped" 
Kahn-Priddy theorem. We also translate our results into Koschorke and Sanderson's geometric framework, yielding relationships among self-intersections of immersions. Sections 3 and 4 contain the proofs of the statements of $\S 1$.

Peter May and Larry Taylor deserve my thanks for stimulating discussions and critical readings of preliminary versions of this work.

1. The compatibility theorems. We review some notation and definitions.

Let $\mathscr{C}_{k}(q)$ be Boardman and Vogt's $[1,11]$ space of ordered $q$ tuples of little cubes disjointly embedded in $I^{k}$. The symmetric group $\Sigma_{q}$ acts freely on $\mathscr{C}_{k}(q)$. Let $B_{k, q}=\mathscr{C}_{k}(q) / \Sigma_{q}$. Note that $B_{\infty, q}$ is a model for the classifying space $B \Sigma_{q}$. If $X$ is a based space, $C_{k} X$ is defined by

$$
C_{k} X=\prod_{q \geqslant 1} \mathscr{C}_{l}(q) \times_{\Sigma_{q}} X^{q} /(\sim)
$$

where $\left[\left(c_{1}, \cdots, c_{q}\right),\left(x_{1}, \cdots, x_{q-1}, *\right)\right] \sim\left[\left(c_{1}, \cdots, c_{q-1}\right),\left(x_{1}, \cdots, x_{q-1}\right)\right]$ generates the equivalence relation. The spaces $C_{k} X$ serve as approximations to $\Omega^{k} \Sigma^{k} X$ in the following sense: there are natural maps $\alpha_{k}: C_{k} X \rightarrow \Omega^{k} \Sigma^{k} X$, preserving all additive structure, which are homotopy equivalences when $X$ is connected, and group completions in general (see $[11,14]$ ).

The space $C_{k} X$ is filtered and the quotients of successive filtrations are the reduced extended power spaces $D_{k, q} X=\mathscr{C}_{k}(q)^{+} \Lambda_{\Sigma_{q}} X^{[q]}$. Here $X^{[q]}$ denotes the $q$-fold smash product and $Y^{+}$denotes the union of a space $Y$ with a disjoint basepoint. Let $C X=C_{\infty} X$ and $D_{q} X=$ $D_{\infty, q} X$.

Let $\eta: \Omega^{k} \Sigma^{k} X \rightarrow \Omega^{k+n} \Sigma^{k+n} X$ be the usual map. There are maps $\eta_{k, n}: C_{k} X \rightarrow \Omega^{n} C_{k} \Sigma^{n} X$ and $\beta_{k, n}: C_{k} X \rightarrow \Omega^{n} C_{k-n} \Sigma^{n} X$ covered by $\eta$ and the identity map respectively; that is, there are commutative diagrams:
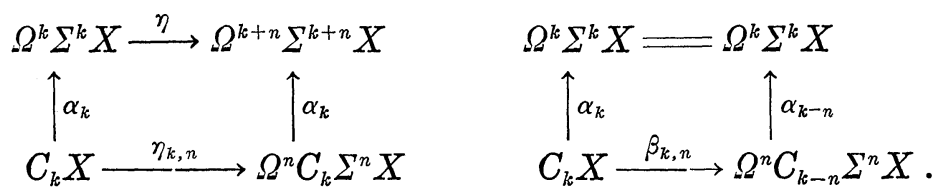

The adjoints, $\widetilde{\eta}_{k, n}$ and $\widetilde{\beta}_{k, n}$ are therefore covered by iterated evaluation maps. Moreover, the adjoints are filtration preserving and thus induce maps $\Delta_{k, n}: \Sigma^{n} D_{k, q} \rightarrow D_{k, q} \Sigma^{n} X$ and $\delta_{k, n}: \Sigma^{n} D_{k, q} X \rightarrow$ $D_{k-n, q} \Sigma^{n} X$. By passage to limits, we obtain maps $\eta_{n}$ and $\beta_{n}: C X \rightarrow$ $\Omega^{n} C \Sigma^{n} X$ and maps $\Delta_{n}$ and $\delta_{n}: \Sigma^{n} D_{q} X \rightarrow D_{q} \Sigma^{n} X$.

We also have maps $i: C_{k} X \rightarrow C_{k+n} X$ and $i: D_{k, q} X \rightarrow D_{k+n, q} X$ induced by the inclusion $I^{k} \rightarrow I^{k+n}$ onto the first $k$ coordinates. 
In [5], F. Cohen, J. P. May, and L. Taylor define James-Hopf maps $j_{q}: C_{k} X \rightarrow C D_{k, q} X$. The space $\prod_{q \geqq 0} Q D_{k, q} X$ is a ring space where $D_{k, 0} X$ is interpreted as $S^{0}$ and the multiplication is induced by natural pairings $\mathscr{C}: D_{k, p} X \wedge D_{k, q} X \rightarrow D_{k, p+q} X$ together with the smash product $\bigoplus: Q Y \times Q Z \rightarrow Q(Y \wedge Z)$. Similarly the space $\Pi_{q \geq 0} C D_{k, q} X$ is a ring space. The James-Hopf maps satisfy a "Cartan formula" [2, 9, 15] and in [2] this is interpreted as the statement that $\Pi_{q \geq 0} j_{q}: C_{k} X \rightarrow$ $\Pi_{q \geq 0} C D_{k, q} X$ is an "exponential" $H$-map taking the additive structure in $C_{k} X$ to the multiplicative one in $\prod_{q \geqq 0} C D_{k, q} X$.

The next proposition is the key to our results.

\section{Proposition 1.1.}

(1) The following diagram commutes.

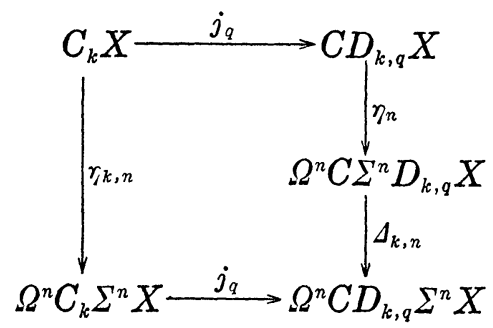

(2) The following diagram homotopy commutes.

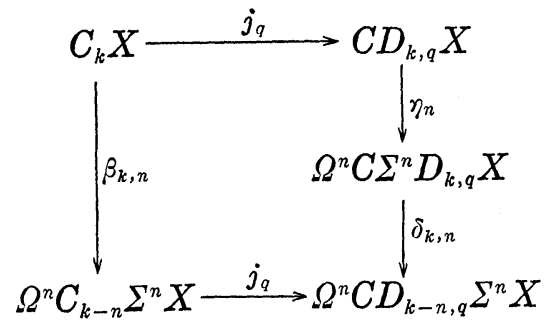

In fact, there is an "exponential" $H$-map

$$
J: C_{k} X \longrightarrow F\left(I^{+}, \Omega^{n} \prod_{q \geq 0} C D_{k-n, q} \Sigma^{n} X\right)
$$

which is a homotopy between $J_{0}=\delta_{k, n} \circ \eta_{n} \circ \prod_{q \geq 0} j_{q}$ and $J_{1}=\prod_{q \geqq 0} j_{q} \circ \beta_{k, n}$.

This will be proved in $\S 4$. Statement (1) follows essentially from a check of the definitions while (2) requires more care.

As shown in [2], the "exponential" properties of the maps $j_{q}$ allow them to be extended to $j_{q}: \Omega^{k} \Sigma^{k} X \rightarrow Q D_{k, q} X$ and $j_{q}: Q X \rightarrow Q D_{q} X$.

THEOREM 1.2. The following diagrams commute up to weak homotopy. 


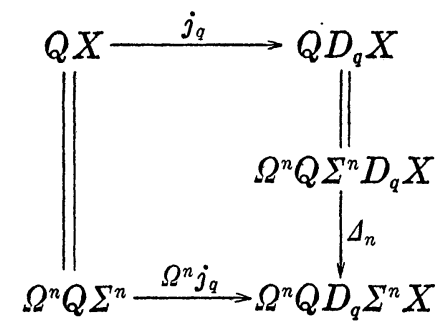

( 2 )

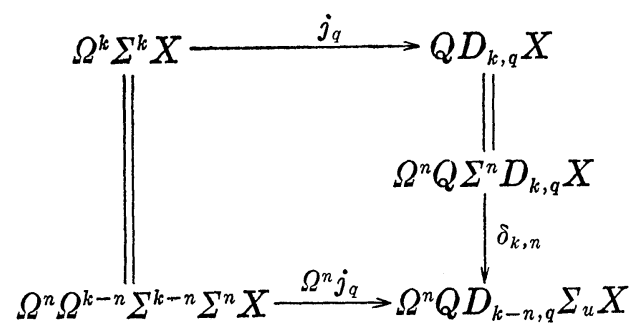

This will be proved in $\S 4$. By weak homotopy, we refer to neglect of $\lim ^{1}$ terms.

Much of the usefulness of the theorem comes from an understanding of the maps $A_{k, n}$ and $\delta_{k, n}$. The next proposition allows us to identify the maps $\delta_{k, n}$ in special cases,

Proposition 1.3.

(1) The composite $\Sigma^{n} D_{n, q} X \stackrel{i}{\rightarrow} \Sigma^{n} D_{k+n, q} X \stackrel{\delta_{k+n, n}}{\longrightarrow} D_{k, q} \Sigma^{n} X$ is nullhomotopic for all $q>1$.

(2) The sequence $\Sigma^{n} D_{n, 2} X \stackrel{i}{\rightarrow} \Sigma^{n} D_{k+n, 2} X \stackrel{\delta_{k+n, n}}{\rightarrow} D_{k, 2} \Sigma^{n} X$ is homotopic to a cofibration sequence. Furthermore, the sequence desuspends $n$ times.

(3) If $p$ is an odd prime, the sequence

$$
\Sigma^{n} D_{n, p} S^{m} \stackrel{i}{\longrightarrow} \Sigma^{n} D_{n+k, p} S^{m} \stackrel{\delta_{k+n, n}}{\longrightarrow} D_{k, p} S^{m+n}
$$

is homotopic to a cofibration sequence, localized at $p$.

When $X=S^{0}, D_{k, 2} X=R P^{k-1^{+}}$and $D_{k, p} X=B_{k, p}^{+}$, hence the proposition has the following consequence.

COROLLARY 1.4. $D_{k, 2} S^{n} \cong \sum^{n} \boldsymbol{R} P^{n+k-1} / \boldsymbol{R} P^{n-1}$ and, localized at an odd prime $p, D_{k, p} S^{n} \cong \Sigma^{n} B_{n+p, k} / B_{n, p}$. Under these identifications, $\delta_{k+n, n}$ can be identified with the projections

$$
\begin{aligned}
& \Sigma^{n} \boldsymbol{R} P^{n+k-1} \longrightarrow \Sigma^{n} \boldsymbol{R} P^{n+k-1} / \boldsymbol{R} P^{n-1} \quad \text { and } \\
& \Sigma^{n} B_{n+k, p} \longrightarrow \Sigma^{n} B_{n+k, p} / B_{n, p} .
\end{aligned}
$$

REMARK 1.5. Proposition 1.3(2) proves and generalizes Milgram's 
Corollary $2.3[13]$ in our setting.

Theorem 1.2 and Corollary 1.4 imply the following.

COROLLARY $1.6 .^{1}$

(1) The following diagram commutes up to weak homotopy.

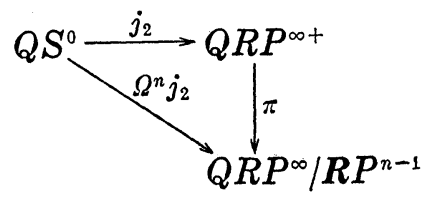

(2) Localized at an odd prime $p$, the following diagram commutes up to weak homotopy.

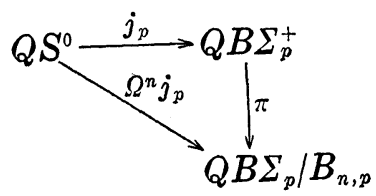

If $n$ is odd then the finiteness of the homotopy groups of $Q \boldsymbol{R} P^{\infty} / \boldsymbol{R} P^{n-1}$ and $Q B \Sigma_{p} / B_{n, p}$ implies that the lim ${ }^{1}$ terms vanish and the diagrams actually homotopy commute.

Finally, the following proposition relates the maps $\Delta_{k, n}$ and $\delta_{k, n}$.

Proposition 1.7. In the following diagram, the quadrilaterals commute and the triangles commute up to homotopy.

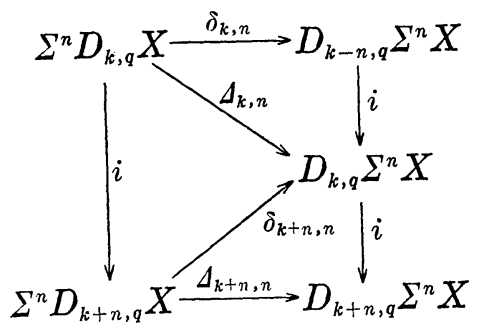

Thus, in the limit as $k$ goes to $\infty, \delta_{n} \cong \Delta_{n}: \Sigma^{n} D_{q} X \rightarrow D_{q} \Sigma^{n} X$.

Propositions 1.3 and 1.7 will be proved in $\S 3$.

2. Applications. In our first application we apply Proposition 1.3 and Theorem 1.2 to the Kahn-Priddy theorem [8]. For a prime $p$, Segal [15] and Caruso, F. Cohen, May, and Taylor [2] find maps

${ }^{1}$ A variant of Corollary 1.6(1) appears in lecture notes by M. Mahowald and A. Unell, Northwestern University, 1977. 
$j: Q_{0} S^{0} \rightarrow Q B \Sigma_{p}$ and $s: Q B \Sigma_{p} \rightarrow Q_{0} S^{0}$ such that the composite is an equivalence localized at $p$. The maps $j$ and $s$ are defined by $j=$ $\pi \circ j_{p}$ and $s=s_{p} \circ i$ in the diagram

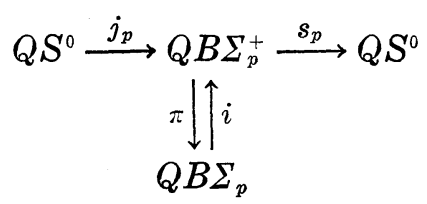

where $j_{p}$ is the James-Hopf map as above and $s_{p}$ is an infinite loop map. Thus $s$ is also an infinite loop map.

Corollary 1.6 implies the following result.

Proposition 2.1. $j$ is a loop map and thus the Kahn-Priddy theorem "deloops" once.

REMARK 2.2. That $j$ does not deloop twice follows from homology calculations in [10].

REMARK 2.3. In [3] it is shown that Proposition 2.1 is precisely what is needed to calculate the order of $\eta: \Omega_{0}^{2 n+1} S^{2 n+1} \rightarrow Q_{0} S^{0}$, localized at 2. From this can be computed the 2 torsion in the order of the bundles $\zeta_{k, q}: F\left(\boldsymbol{R}^{k}, q\right) \times_{\Sigma_{q}} \boldsymbol{R}^{q} \rightarrow F\left(\boldsymbol{R}^{k}, q\right) / \Sigma_{q}$. Note that the Thom complex of $n \zeta_{k, q}$ is precisely the space $D_{k, q} S^{n}$.

Our next application mixes our compatibility statements with the $\mathrm{E}-\mathrm{H}-\mathrm{P}$ sequence. We need the following version of Milgram's generalized E-H-P sequence.

LEMMA 2.4 [12].

If $X$ is $m-1$ connected (and $\Sigma^{n} X$ is connected) then

$$
\Omega^{n} \Sigma^{n} X \stackrel{\eta}{\longrightarrow} Q X \stackrel{\Omega^{n} j_{2}}{\longrightarrow} \Omega^{n} Q D_{2} \Sigma^{n} X
$$

is homotopic to a fibration through dimensions $<2 n+3 m-1$ (the metastable range).

The corresponding long exact sequence of homotopy groups is known as the generalized E-H-P sequence.

Corollary 2.5. If $X$ is $m-1$ connected then, through dimensions $<2 n+3 m-1$, the following is a homotopy pullback diagram.

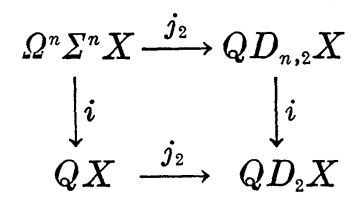


In particular, through dimensions $<2 n-1$, the following diagram is a pullback.

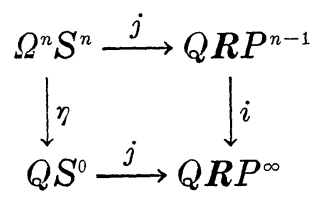

Thus the desuspension of 2-torsion through the metastable range is detected by the stable map $i$.

Proof. By Theorem 1.2, the following diagram commutes

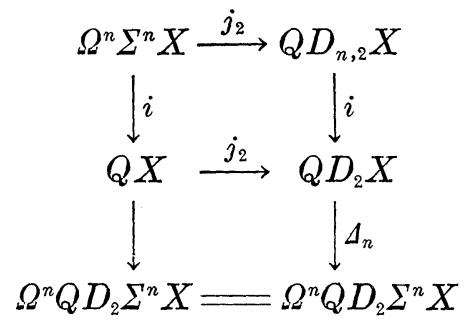

Proposition 1.3 implies that the right hand vertical sequence is a fibration and the lemma says that the left hand vertical sequence is a fibration through the desired dimension.

Finally we relate our results to Koschorke and Sanderson's theory of self intersections of immersions [9].

Using the notation of [9], let $V$ be a manifold without boundary and let $\xi$ be a vector bundle over a space $B$, Let $\mathscr{J}_{k}(V, \xi)$ be the bordism set of embeddings $g=\left(g_{1}, g_{2}\right): M \rightarrow V \times \boldsymbol{R}^{k}$ for which $g_{1}: M \rightarrow V$ is an immersion with normal bundle $\nu$ expressed as a pullback $\bar{g}: \nu \rightarrow$ $\xi$. Thus an element of $\mathcal{F}_{k}(V, \xi)$ is represented by a triple $(M, g, \bar{g})$. By abuse of notation, we will denote this class by $[M]$.

Let $V_{c}$ denote the one point compactification of $V$. Let $T(\xi)$ denote the Thom space of $\xi$.

Proposition 2.6 [9]. There is a bijection

$$
\mathscr{J}_{k}(V, \xi)=\left[V_{c}, C_{k} T(\xi)\right]
$$

This is proved using a generalization of the classical ThomPontrjagin construction. See [9] for details.

To describe the geometric operations, we recall the following definition. Given a self transverse immersion $f: M \rightarrow V$, let $\tilde{M}(q)=$ $\left\{\left(x_{1}, \cdots, x_{q}\right) \in M^{q}: f\left(x_{1}\right)=\cdots=f\left(x_{q}\right)\right.$, and $x_{i} \neq x_{j}$ if $\left.i \neq j\right\}$. This is a closed submanifold of $M^{q}$ on which $\Sigma_{q}$ acts freely. Let $M(q)=$ $\widetilde{M}(q) / \Sigma_{k}$, called the $q$-tuple point manifold. 
If $\xi$ is a bundle over $B$, let $\xi_{k, q}$ be the twisted power bundle over $\mathscr{C}_{k}(q) \times_{\Sigma_{q}} B^{q}$. Note that $T\left(\xi_{k, q}\right)=D_{k, q} T(\xi)$. Let $\varepsilon^{n}$ denote the trivial bundle of dimension $n$.

Koschorke and Sanderson define operations $\theta_{q}: \mathscr{J}_{k}(V, \xi) \rightarrow \mathscr{J}_{\infty}\left(V, \xi_{k, q}\right)$, roughly defined by $\theta_{q}[M]=[M(q)]$. These operations are related to the James-Hopf maps as follows.

Proposition 2.7 [9]. There is a commutative diagram

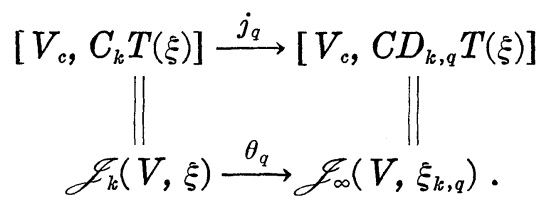

Proposition 1.1 now has following consequences.

Proposition 2.8. The following diagrams commute.

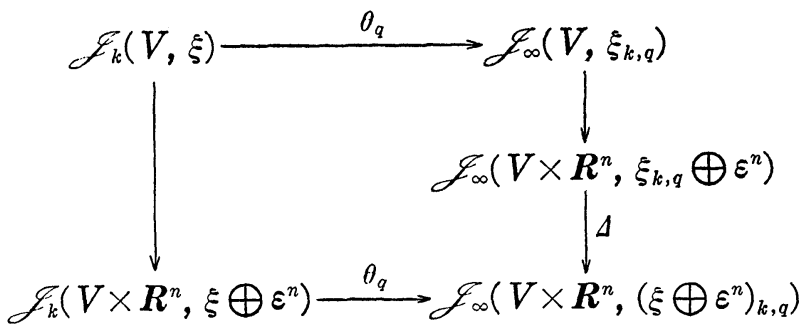

Here $\Delta$ is induced by the diagonal inclusion of vector bundles:

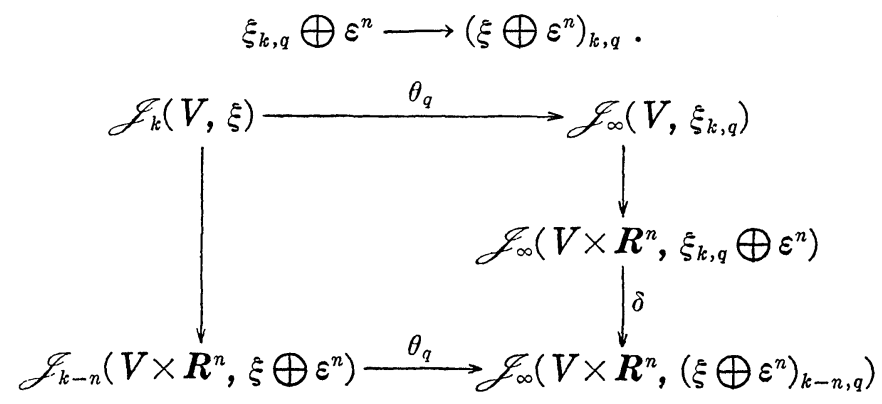

Here $\delta$ is induced by $\operatorname{map} T\left(\xi_{k, q} \oplus \varepsilon^{n}\right) \rightarrow T\left(\left(\xi \oplus \varepsilon^{n}\right)_{k-n, q}\right)$.

REMARK 2.9. To understand the map $\Delta$ geometrically, note that any bundle inclusion $f: \xi \rightarrow \eta$ of bundles over $B$ induces a map $f_{*}: \mathscr{J}_{m}(V, \xi) \rightarrow \mathscr{J}_{m}(V, \eta)$ which can be interpreted as follows. Given $g: M \rightarrow V \times \boldsymbol{R}^{m}$ and $\bar{g}: \nu \rightarrow \xi$, the composite $f \bar{g}: \nu \rightarrow \eta$ can be approximated by a map $h$ transverse to the zero section of $\eta$. Then $f_{*}[M]=\left[h^{-1}(B)\right]$. Note that $h^{-1}(B)$ is a submanifold of some manifold 
$M^{\prime} \in[M]$.

Proposition 1.3(2) together with Proposition 2.6, yields the next result. This is just a long exact sequence associated to the fibration:

$$
Q T\left(\xi_{n, 2} \oplus \varepsilon^{n}\right) \longrightarrow Q T\left(\xi_{n+k, 2} \oplus \varepsilon^{n}\right) \longrightarrow Q T\left(\left(\xi \oplus \varepsilon^{n}\right)_{k, 2}\right) \text {. }
$$

Proposition 2.10. The following sequence is exact.

$$
\begin{aligned}
& \longrightarrow \mathscr{F}_{\infty}\left(V \times \boldsymbol{R}, \xi_{k+n, 2} \oplus \varepsilon^{n}\right) \longrightarrow \mathscr{J}_{\infty}\left(V \times \boldsymbol{R},\left(\xi \oplus \varepsilon^{n}\right)_{k, 2}\right) \\
& \longrightarrow \mathscr{J}_{\infty}\left(V, \xi_{n, 2} \oplus \varepsilon^{n}\right) \longrightarrow \mathscr{J}_{\infty}\left(V, \xi_{k+n, 2} \oplus \varepsilon^{n}\right) \stackrel{\delta}{\longrightarrow} \mathscr{J}_{\infty}\left(V,\left(\xi \oplus \varepsilon^{n}\right)_{k, 2}\right) .
\end{aligned}
$$

3. The maps $A_{k, n}$ and $\delta_{k, n}$. We first define the maps $\eta_{k, n}: C_{k} X \rightarrow$ $\Omega^{n} C_{k} \Sigma^{n} X$ and $\beta_{k, n}: C_{k} X \rightarrow \Omega^{n} C_{k-n} \Sigma^{n} X$ used in $\S 1$. Let $\eta_{k, n}: C_{k} X \rightarrow$ $\Omega^{n} C_{k} \Sigma^{n} X$, with adjoint $\tilde{\eta}_{k, n}$, be defined by

$$
\tilde{\eta}_{k, n}\left(\left[\left(c_{1}, \cdots, c_{q}\right),\left(x_{1}, \cdots, x_{q}\right), t\right]\right)=\left[\left(c_{1}, \cdots, c_{q}\right),\left(\left(x_{1}, t\right), \cdots,\left(x_{q}, t\right)\right)\right]
$$

where $t \in S^{n},\left(c_{1}, \cdots, c_{q}\right) \in \mathscr{C}_{k}(q)$, and $\left(x_{1}, \cdots, x_{q}\right) \in X^{q} . \quad \beta_{k, 1}$ is the map defined by May in [11, Prop. 5.4].

It is useful to view $\beta_{k, 1}$ in the following way. Let $c_{i}=c_{i}^{\prime} \times c_{i}^{\prime \prime}$ where $c_{i}^{\prime}: I \rightarrow I, c_{i}^{\prime \prime}: I^{k-1} \rightarrow I^{k-1}$. Then $\beta_{k, 1}\left(\left[\left(c_{1}, \cdots, c_{q}\right),\left(x_{1}, \cdots, x_{q}\right)\right]\right)(t)=$ $\left[\left(c_{i}^{\prime \prime}, \cdots, c_{q}^{\prime \prime}\right),\left(\left(x_{1}, s_{1}(t)\right), \cdots,\left(x_{q}, s_{q}(t)\right)\right)\right]$ where $s_{i}(t)$ is defined by $c_{i}^{\prime}\left(s_{i}(t)\right)=$ $t$ if $t \in \operatorname{Im} c_{i}^{\prime}$ and

$$
s_{i}(t)=\left\{\begin{array}{lll}
0 & \text { if } \quad t<\operatorname{Im} c_{i}^{\prime} \\
1 & \text { if } \quad t>\operatorname{Im} c_{i}^{\prime}
\end{array} .\right.
$$

This definition makes sense because if the cubes $\left\{c_{i}^{\prime}, \cdots, c_{q}^{\prime \prime}\right\}$ are not all disjoint then exactly the right number of pairs $\left(x_{i}, s_{i}(t)\right) \in \Sigma X$ are the basepoint, by virtue of $s_{i}(t)=0$ or 1 .

Iteration defines maps $\beta_{k, n} ; C_{k} X \rightarrow \Omega^{n} C_{k-n} \Sigma^{n} X$, with adjoint $\widetilde{\beta}_{k, n}$. As mentioned before, $\widetilde{\eta}_{k, n}$ and $\widetilde{\beta}_{k, n}$ are filtration preserving and thus induce $\Delta_{k, n}$ and $\delta_{k, n}$. Recall that $i: C_{k} X \rightarrow C_{k+n} X$ is the map induced by the inclusion $I^{k} \rightarrow I^{k+n}$ onto the first $k$ coordinates. We will later make use of the fact that $i$ is homotopic (by a filtration preserving homotopy) to the map $i^{\prime}: C_{k} X \rightarrow C_{k+n} X$ induced by the inclusion $I^{k} \rightarrow$ $I^{k+n}$ onto the last $k$ coordinates. For a proof of this, see [11, Lemma $4.9]$.

Proof of Proposition 1.3. In statements (1), (2), and (3) we first reduce the general case to the case $n=1$.

Consider the following commutative diagram.

Inductively, if we assume statement (1) for $n-1$, then the statement follows for $n$ by consideration of the two vertical sequences.

Next, suppose $q=2$ in the diagram. If we assume that statement 


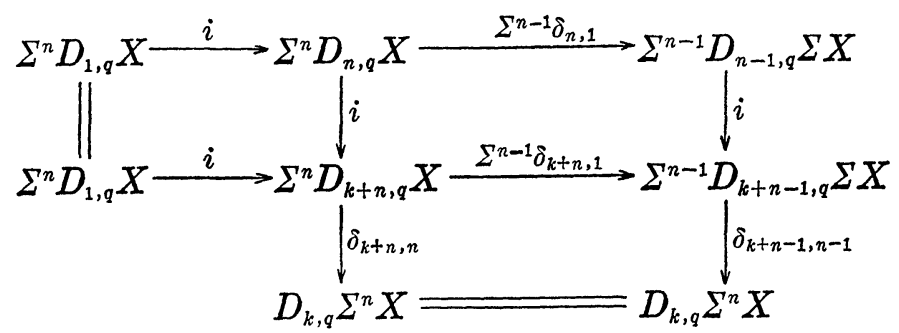

(2) of the proposition is true for $n=1$, then the horizontal sequences are cofibrations, and inductively we assume that the right vertical sequence is a cofibration. Then, by diagram chasing, the left vertical sequence will be a cofibration.

Statement (3) reduces to the case $n=1$ in a similar fashion, upon letting $q=p$ in the diagram. Note that the argument given above for statement (2) goes through here because the set of spheres is closed under suspension.

Thus to prove statement (1) we just need to show that the composite $\Sigma D_{1, q} X \stackrel{i}{\rightarrow} \Sigma D_{k+1, q} X \stackrel{\delta_{k+1,1}}{\longrightarrow} D_{k, q} \Sigma X$ is nullhomotopic for $q>1$. This follows immediately from the following observation.

LEMmA 3.1. The composite $\Sigma C_{1} X \stackrel{i}{\rightarrow} \Sigma C_{k+1} X \stackrel{\beta_{k+1,1}}{\longrightarrow} C_{k} \Sigma X$ has image in filtration 1.

To prove statement (2) we need to show that the following sequence is homotopic to a cofibration sequence.

$$
\Sigma D_{1,2} X \stackrel{i}{\longrightarrow} \Sigma D_{k+1,2} X \stackrel{\delta_{k+1,1}}{\longrightarrow} D_{k, 2} \Sigma X .
$$

This sequence is equivalent to the following.

$$
\begin{aligned}
S^{0^{+}} \Lambda_{z_{2}}(X \wedge X) \wedge S^{1} \longrightarrow & S^{k^{+}} \Lambda_{z_{2}}(X \wedge X) \wedge S^{1} \\
& \longrightarrow S^{k-1+} \Lambda_{z_{2}}(\Sigma X \wedge \Sigma X)
\end{aligned}
$$

Here $(\Sigma X \wedge \Sigma X)$ is equivariantly homeomorphic to $(X \wedge X) \wedge\left(S^{1} \wedge S^{1}\right)$ where all spaces have the obvious permutation actions. $\left(S^{1} \wedge S^{1}\right)$ is then equivalent to $S^{1} \wedge S^{1}$ where $S^{1}$ has the trivial action and $\boldsymbol{S}^{1}=[-1,1] /-1 \sim 1$ with $Z_{2}$ action given by $t \rightarrow-t$.

Let points in $S^{k}$ be benoted by $\left(s_{0}, \cdots, s_{k}\right) \in \boldsymbol{R}^{k+1}$ with $\Sigma s_{i}^{2}=1$. Then our sequence desuspends to

$$
S^{0+} \Lambda_{z_{2}}(X \wedge X) \stackrel{i}{\longrightarrow} S^{k^{+}} \Lambda_{z_{2}}(X \wedge X) \stackrel{p}{\longrightarrow} S^{k-1^{+}} \bigwedge_{z_{2}} S^{1}(X \wedge X)
$$

where 
$p\left(s_{0}, \cdots, s_{k}, x_{1}, x_{2}\right)=\left\{\begin{array}{l}\left(\frac{s_{1}}{\sqrt{1-s_{0}^{2}}}, \cdots, \frac{s_{k}}{\sqrt{1-s_{0}^{2}}}, s_{0}, x_{1}, x_{2}\right) \text { if }\left|s_{0}\right|<1 \\ * \text { otherwise }\end{array}\right.$ and $i$ is defined by $i: S^{0} \rightarrow S^{k}$ with $i( \pm 1)=( \pm 1,0,0, \cdots, 0)$. Then $p$ is homeomorphism of pairs,

$$
p:\left(S^{k} \Lambda_{z_{2}}(X \wedge X), S^{0+} \Lambda_{z_{2}}(X \wedge X)\right) \cong\left(S^{k-1+} \Lambda_{z_{2}} S^{1} \wedge(X \wedge X), *\right)
$$

with inverse given by

$$
\left(s_{1}, \cdots, s_{k}, t, x_{1}, x_{2}\right) \longmapsto\left(t, \sqrt{\left(1-t^{2}\right)} s_{1}, \cdots, \sqrt{\left(1-t^{2}\right)} s_{k}, x_{1}, x_{2}\right) .
$$

REMARK 3.2. This last proof is essentially a generalization of the standard argument that the Thom space of $m \xi_{k}, m$ copies of the canonical line bundle $\xi_{k}$ over $\boldsymbol{R} \boldsymbol{P}^{k}$, is homeomorphic to $\boldsymbol{R} \boldsymbol{P}^{m+k} / \boldsymbol{R} \boldsymbol{P}^{m-1}$. See [6, Example 15.1.7].

Finally, to prove statement (3) of Proposition 1.3, in the case $n=1$, we need to show that the sequence

$$
S^{p m+1} \stackrel{i}{\longrightarrow} \Sigma D_{k+1, p} S^{m} \stackrel{\delta_{k+1,1}}{\longrightarrow} D_{k, p} S^{m+1}
$$

is a cofibration when localized at $p$. By virtue of statement (1), it suffices to show that the sequence acts homologically as a cofibration. Recall that $\delta_{k+1,1}$ is induced from the map $\widetilde{\beta}_{k, 1}: \Sigma C_{k+1} S^{m} \rightarrow C_{k} S^{m+1}$ which by definition induces the homology suspension $\sigma_{*}$. Let $Q^{i}$ denote a Dyer-Lashof operation, let $\beta$ denote the Bockstein, and let $\lambda_{k}(x, y)$ denote the Browder operation on the homology of a $\mathscr{C}_{k+1}$-space (see [4] for the definitions). In [4] it is shown that $\sigma_{*} Q^{i} x=Q^{i} \sigma_{*} x$ and $\sigma_{*} \lambda_{k}(x, y)=\lambda_{k-1}\left(\sigma_{*} x, \sigma_{*} y\right)$; while $\sigma_{*} \beta=\beta \sigma_{*}$ up to sign. We conclude that the above sequence induces a short exact homology sequence:

$0 \longrightarrow H_{*}\left(S^{p m+1} ; Z_{p}\right) \stackrel{i_{*}}{\longrightarrow} H_{*}\left(\Sigma D_{k+1, p} S^{m} ; Z_{p}\right) \stackrel{\delta_{k+1,1 *}}{\longrightarrow} H_{*}\left(D_{k, p} S^{m+1} ; Z_{n}\right) \longrightarrow 0$.

The proposition follows.

Proof of Proposition 1.7. It suffices to show that the quadrilaterals commute and the triangles commute up to a natural filtration preserving homotopy in the following diagram.

The quadrilaterals can be seen to commute by a check of definitions.

For the commutativity of the triangles it suffices to let $n=1$. The left hand triangle commutes when $i$ is replaced by $i^{\prime}: C_{k} X \rightarrow$ $C_{k+1} X$, induced by the inclusion $\boldsymbol{R}^{k} \rightarrow \boldsymbol{R}^{k+1}$ onto the last $k$ coordinates of $\boldsymbol{R}^{k+1}$. As mentioned before, $i$ is homotopic to $i^{\prime}$. To prove that 


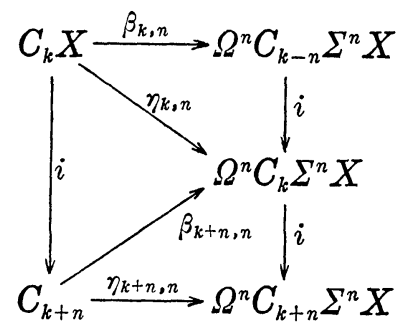

the right hand triangles commute, we show that

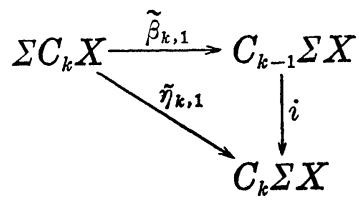

commutes up to a filtration preserving homotopy. Again replace $i$ by $i^{\prime}$.

If $[c, x] \in C_{k} X$ with $c=\left(c_{1}^{\prime} \times c_{1}^{\prime \prime}, \cdots, c_{q}^{\prime} \times c_{q}^{\prime \prime}\right)$ and $x=\left(x_{1}, \cdots, x_{q}\right)$ then we have following formulae:

$$
\begin{aligned}
i^{\prime} \circ \widetilde{\beta}_{k, 1}(([c, x], t)) & =\left[\left(1 \times c_{1}^{\prime \prime}, \cdots, 1 \times c_{q}^{\prime \prime}\right),\left(\left(x_{1}, s_{1}(t)\right), \cdots,\left(x_{q}, s_{q}(t)\right)\right)\right] \\
\tilde{\eta}_{k, 1}(([c, x], t)) & =\left[\left(c_{1}^{\prime} \times c_{1}^{\prime \prime}, \cdots, c_{q}^{\prime} \times c_{q}^{\prime \prime}\right),\left(\left(x_{1}, t, \cdots,\left(x_{q}, t\right)\right)\right)\right] .
\end{aligned}
$$

To show that these are homotopic, we show that maps are homotopic to the map $h: \Sigma C_{k} X \rightarrow C_{k} \Sigma X$ defined by

$$
h(([c, x], t))=\left[\left(c_{1}^{\prime} \times c_{1}^{\prime \prime}, \cdots, c_{q}^{\prime} \times c_{q}^{\prime \prime}\right),\left(\left(x_{1}, s_{1}(t)\right), \cdots,\left(x_{q}, s_{q}(t)\right)\right)\right] .
$$

First, $\tilde{\eta}_{k, 1} \cong h$ by shrinking id: $I \rightarrow I$ to $s_{i}: I \rightarrow I$ in some natural way (continuous in $c_{i}^{\prime}$ ). Second, $h \cong i^{\prime} \circ \widetilde{\beta}_{k, 1}$ by expanding each $c_{i}^{\prime}$ to id: $I \rightarrow I$ in a natural way.

4. The James maps and the compatibility theorems. In this section we prove Proposition 1.1 and Theorem 1.2.

We first note that a straightforward inductive argument reduces our statements to the case $n=1$.

Proof of Proposition 1.1. The commutativity of diagram (1) in its adjoint form follows from a straightforward check of definitions.

We digress to note that this diagram is a special case of the following more general statement about maps $j_{q}$. We use the language of coefficient systems and $\Pi$-spaces from [5].

In general, there are James maps 


$$
j_{q}: C X \longrightarrow C^{\prime}\left(D_{q}(\mathscr{C}, \boldsymbol{X})\right)
$$

where $X$ is a $\Pi$-space and $\mathscr{C}$ and $\mathscr{C}^{\prime}$ are coefficient systems with a James system $\left\{\xi_{q r}\right\}: \mathscr{C} \rightarrow \mathscr{C}^{\prime}$. Abbreviate $D_{q}(\mathscr{C}, \boldsymbol{X})$ to $D_{q} \boldsymbol{X}$.

LEMMA 4.1. For any based space $P$, the diagonal maps $\Delta: P \rightarrow P^{k}$ induce following commutative diagram.

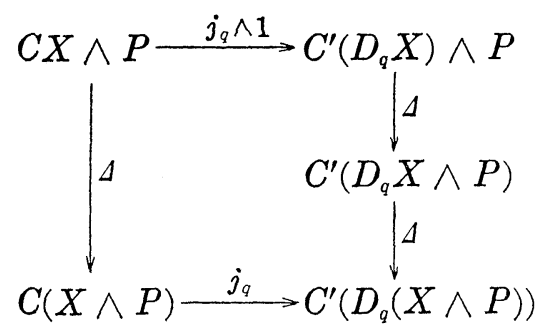

To see the specialization of this diagram to the adjoint of diagram (1) of Proposition 1.1 and to prepare for proof of (2), we recall the construction of the James-Hopf maps from [5].

Let $F(Y, q) \subset Y^{q}$ denote the configuration space of distinct $q$-tuples in $Y^{q}$. If $X$ is based, let

$$
C(Y, X)=\prod_{q \geqq 1} F(Y, q) \times_{\Sigma_{q}} X^{q} /(\sim)
$$

with the equivalence relation generated as before. There is a natural equivalence $C_{k} X \cong C\left(\boldsymbol{R}^{k}, X\right)$, under the map which assigns to a set of cubes, their centers.

The James-Hopf maps are constructed as follows. We first define maps $j_{q}: C_{k} X \rightarrow C\left(B_{k, q}, D_{k, q} X\right)$. If $[c, X] \in C_{k} X$ is in filtration $r$, then $j_{q}((c, x))$ will be in filtration $m$, where $m$ is the binomial coefficient $\left(\begin{array}{c}r \\ q\end{array}\right)$. Let $\left\{A_{1}, \cdots, A_{m}\right\}$ be the $m$ subsets of $c$ of cardinality $q$. There are corresponding subsets $\left\{B_{1}, \cdots, B_{m}\right\}$ of $x$. Let

$$
j_{q}([c, x])=\left[\left(A_{1}, \cdots, A_{m}\right),\left(\left(A_{1}, B_{1}\right), \cdots,\left(A_{m}, B_{m}\right)\right)\right] .
$$

This is well-defined and continuous.

An embedding $B_{k, q} \rightarrow \boldsymbol{R}^{\infty}$ induces a map

$$
C\left(B_{k, q}, D_{k, q} X\right) \longrightarrow C\left(R^{\infty}, D_{k, q} X\right),
$$

and the composite with the above maps defines $j_{q}: C_{k} X \rightarrow C D_{k, q} X$.

Proposition 1.1 (1) now follows from the application of the lemma to the special case in which $\mathscr{C}=\mathscr{C}_{k}$, the little cubes operad, $\mathscr{C}^{\prime}=\mathscr{C}\left(B_{k, q}\right)$, the coefficient system with $t$ th space $F\left(B_{k, q}, t\right)$, and $P=S^{1}$.

The proof of statement (2) is a bit more delicate. We recall 
that an embedding $B_{k, q} \subset \boldsymbol{R}^{\infty}$ is involved in defining the maps $j_{q}$. We can make these compatible as $k$ varies by chossing a fixed embedding $B \Sigma_{q} \rightarrow \boldsymbol{R}^{\infty}$ and then embedding $B_{k, q}$ via the composite $B_{k, q} \rightarrow B \Sigma_{q} \rightarrow R^{\infty}$. Then $j_{q}: C_{k} X \rightarrow C\left(\boldsymbol{R}^{\infty}, D_{k, q} X\right)$ factors as the composite $C_{k} X \rightarrow C\left(B_{k, q}, D_{k, q} X\right) \rightarrow C\left(B \Sigma_{q}, D_{k, q} X\right) \rightarrow C\left(\boldsymbol{R}^{\infty}, D_{k, q} X\right)$. The space $\prod_{q \geqq 0} C\left(B_{k, q}, D_{k, q} X\right)$ has an $H$-space structure induced from the maps $B_{k, q} \times B_{k, p} \rightarrow B_{k, q+p}$ and $D_{k, q} X \wedge D_{k, p} X \rightarrow D_{k, q+p} X$ [2]. It suffices to show that the following diagram commutes, and that, when the product is taken over all $q \geqq 0$, the homotopy is through compatible $H$-maps.



To show this we make use auxiliary spaces, lying between $\mathscr{C}_{k-1}(q)$ and $\mathscr{C}_{k}(q)$, which also form a coefficient system. Let $\mathscr{C}_{k}^{\prime}(q)=$ ordered sets of $q$ little $k$-cubes $\left(c_{1}, \cdots, c_{q}\right)$ such that $\left(c_{1}^{\prime \prime}, \cdots, c_{q}^{\prime \prime}\right)$ have disjoint images, where $c_{i}=c_{i}^{\prime} \times c_{i}^{\prime \prime}, c_{i}^{\prime}: I \rightarrow I, c_{i}^{\prime \prime}: I^{k-1} \rightarrow I^{k-1}$. Let $B_{k, q}^{\prime}=$ $\mathscr{C}_{k}^{\prime}(q) / \Sigma_{q}$.

There is a projection map $\beta: \mathscr{C}_{k}^{\prime}(q) \rightarrow \mathscr{C}_{k-1}(q)$ defined by

$$
\beta\left(\left(c_{1}, \cdots, c_{q}\right)\right)=\left(c_{1}^{\prime \prime}, \cdots, c_{q}^{\prime \prime}\right) .
$$

Let $i_{1}: \mathscr{C}_{k-1}(q) \rightarrow \mathscr{C}_{k}^{\prime}(q)$ be the inclusion map defined by $i_{1}\left(c_{1}, \cdots, c_{q}\right)=$ $\left(1 \times c_{1}, \cdots, 1 \times c_{q}\right)$ and let $i_{2}: \mathscr{C}_{k}^{\prime}(q) \rightarrow \mathscr{C}_{k}(q)$ be the obvious inclusion. Then $\beta \circ i_{1}=i d_{\mathscr{G}_{k-1}(q)}$ and $i_{1} \circ \beta \cong i d_{\mathscr{\sigma}_{k}^{\prime}(q)}$ equivariantly via a homotopy $H_{t}: \mathscr{C}_{k}^{\prime}(q) \rightarrow \mathscr{C}_{k}^{\prime}(q)$ "stretching" the $c_{i}^{\prime}$ to $i d: I \rightarrow I$ in a natural way. Consider the following diagram. Once again, note that $i$ has replaced by the homotopic map $i^{\prime}$.

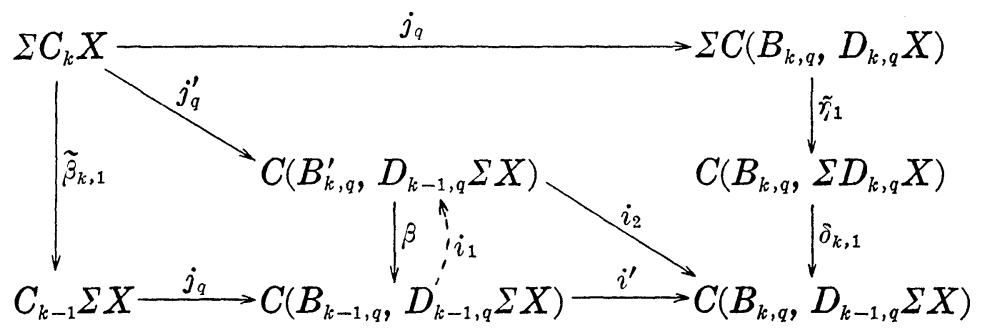

By inspection, $\delta_{k, 1} \circ \tilde{\eta}_{1} \circ j_{q}$ factors through $C\left(B_{k, q}^{\prime}, D_{k-1, q} \Sigma X\right)$, thus defining the map $j_{q}^{\prime}$. Thus the upper part of the diagram commutes. By a 
check of definitions, one can see that the left hand quadralateral commutes, i.e., $\beta \circ j=j_{q} \circ \widetilde{\beta}_{k, 1}$.

Finally we check that the triangle commutes up to homotopy. $i^{\prime}=$ $i_{2} \circ i_{1}$ so that $i^{\prime} \circ \beta=i_{2} \circ i_{1} \circ \beta \cong i_{2} \circ 1=i_{2}$ Thus diagram (2) of Proposition 1.1 commutes up to homotopy.

To conclude that this homotopy is an $H$-map we first note that $\Pi_{q \geq 0} C\left(B_{k, q}^{\prime}, D_{k-1, q} X\right)$ also has a natural $H$-space structure. In the language of [2], $\mathscr{C}^{\prime}$ has a separated sum. Proposition 1.1 (2) now follows from the following lemma.

LEMMA 4.2. The following maps are strict $H$-maps.

(a) $\prod_{q \geqq 0} j_{q}: C_{k} X \rightarrow \prod_{q \geqq 0} C\left(B_{k, q}, D_{k, q} X\right)$

(b) $\beta_{k, 1}: C_{k} X \rightarrow \Omega C_{k-1} \Sigma X$

(c) $i_{1}: \prod_{q \geqq 0} C\left(B_{k-1, q}, D_{k-1, q} \Sigma X\right) \rightarrow \prod_{q \geq 0} C\left(B_{k, q}, D_{k-1, q} \Sigma X\right)$

(d) $i_{2}: \prod_{q \geq 0} C\left(B_{k, q}^{\prime}, D_{k-1, q} \Sigma X\right) \rightarrow \prod_{q \geq 0} C\left(B_{k, q}, D_{k-1, q} \Sigma X\right)$

(e) $H: \prod_{q \geq 0} C\left(B_{k, q}^{\prime}, D_{k-1,1 q} \Sigma X\right) \rightarrow F\left(I^{+}, \prod_{q \geq 0} C\left(B_{k, q}^{\prime}, D_{k-1, q} \Sigma X\right)\right)$

(f) The homotopy between $i$ and $i^{\prime}: \prod_{q \geq 0} C\left(B_{k-1, q}, D_{k-1, q} \Sigma X\right) \rightarrow$ $\Pi_{q \geqq 0} C\left(B_{k, q}, D_{k-1, q} \Sigma X\right)$

(g) The composite

$$
\prod_{q \geq 0} C\left(B_{k, q}, D_{k, q} X\right) \stackrel{\eta_{1}}{\longrightarrow} \Omega \prod_{q \geq 0} C\left(B_{k, q}, \Sigma D_{k, q} X\right) \stackrel{\delta_{k, 1}}{\longrightarrow} \Omega \prod_{q \geq 0} C\left(B_{k, q}, D_{k-1, q} \Sigma X\right)
$$

(h) $\prod_{q \geq 0} j_{q}^{\prime}: C_{k} X \rightarrow \prod_{q \geq 0} C\left(B_{k, q}^{\prime}, D_{k-1, q} \Sigma X\right)$.

That (a) is a strict $H$-map is shown in [2]. That the maps (b)(g) are strict $H$-maps follows from a straightforward check of the definitions. Statement (h) follows from statements (a), (d), and (g) and the fact that $i_{2}$ is an injection.

Proof of Theorem 1.2. As remarked before, it suffices to prove the theorem when $n=1$. We will just show that the second diagram homotopy commutes; that the first diagram commutes follows from a slightly simpler but essentially identical argument.

To extend the James-Hopf maps $j_{q}: C_{k} X \rightarrow C D_{k, q} X \rightarrow Q D_{k, q} X$ to $j_{q}: \Omega^{k} \Sigma^{k} X \rightarrow Q D_{k, q} X$ we note that $\prod_{q \geqq 0} j_{q}: C_{k} X \rightarrow \prod_{q \geqq 0} Q D_{k, q} X$ is an $H$-map and that $\prod_{q \geq 0} Q D_{k, q} X$ is grouplike. A universal property of group completions [2] then allows $\Pi_{q \geq 0} j_{q}$ to be extended to $\Omega^{k} \Sigma^{k} X$, uniquely up to weak homotopy, i.e., disregrading $\lim ^{1}$ terms.

Similarly the homotopy $J: C_{k} X \rightarrow F\left(I^{+}, \Omega \prod_{q \geqq 0} Q D_{k-1, q} \Sigma X\right)$ of Proposition 1.1 extends, uniquely up to weak homotopy, to give a homotopy of weak $H$-maps $\widetilde{J}: \Omega^{k} \Sigma^{k} X \rightarrow F\left(I^{+}, \Omega \prod_{q \geq 0} Q_{k-1, q} \Sigma X\right)$. Then $\widetilde{J}_{1}=\Omega \prod_{q \geq 0} j_{q}$ because $\Omega \prod_{q \geq 0} j_{q}$ does cover $J_{1}$ and is an $H$-map. As before, $\delta_{k, 1}: \prod_{q \geq 0} Q D_{k, q} X \rightarrow \prod_{q \geq 0} Q D_{k-1, q} \Sigma X$ is an $H$-map and thus so also is $\delta_{k, 1} \circ \prod_{q \geqq 0} j_{q}$. This composite covers $J_{0}$ and thus $\widetilde{J}_{0}=\delta_{k, 1} \circ \prod_{q \geqq 0} j_{q}$. 


\section{Theorem 1.2 follows.}

\section{REFERENCES}

1. J. M. Boardmen and R. M. Vogt, Homotopy-everything H-spaces, Bull. Amer. Math. Soc., 74 (1968), 1117-1122.

2. J. Caruso, F. R. Cohen, J. P. May and L. R. Taylor, James maps, Segal maps and the Kahn-Priddy theorem, to appear.

3. F. R. Cohen, R. L. Cohen, N. J. Kuhn and J. A. Neisendorfer, Bundles over configuration spaces, to appear.

4. F. R. Cohen, T. J. Laba and J. P. May, The Homology of Iterated Loop Spaces, Springer Lecture Notes in Mathematics, Vol. 533, 1976.

5. F. R. Cohen, J. P. May and L. R. Taylor, Splitting of certain spaces $C X$, Proc. Camb. Phil. Soc., 84 (1978), 465-496.

6. D. Husemoller, Fibre Bundles, Springer-Verlag. 1966.

7. I. M. James, Reduced product spaces, Annals of Math., 62 (1955), 170-197.

8. D. S. Kahn and S. B. Priddy, Applications of the transfer to stable homotopy theory, Bull. Amer. Math. Soc., 76 (1972), 981-987.

9. U. Koschorke and B. Sanderson, Self-intersections and higher Hopf invariants, Topology, 17 (1978); 283-290.

10. N. J. Kuhn, The homology of the James-Hopf maps, Illinois J. Math., to appear. 11. J. P. May, The Geometry of Iterated Loop Spaces, Springer Lecture Notes in Mathematics, Vol. 271, 1972.

12. - Infinite loop space theory, Bull. Amer. Math. Soc., 83, no. 4 (1977), 456-494.

13. R. J. Milgram, Unstable Homotopy from the Stable Point of View, Springer Lecture Notes in Mathematics, Vol. 368, 1974.

14. G. Segal, Configuration spaces and iterated loop spaces, Invent. Math., 21 (1973), $213-221$.

15. Operations in stable homotopy, London Math. Soc. Lecture Notes no. 11, $105-110$.

16. V. P. Snaith, A stable decomposition for $\Omega^{n} \Sigma^{n} X$, J. London Math. Soc., 7 (1974), $577-583$.

17. H. Toda, Composition Methods in the Homotopy Groups of Spheres, Princeton University Press, 1962.

Received December 10, 1980.

UNIVERSITY OF WASHINGTON

Seattle, WA 98195

Current address: Princeton University

Princeton, NJ 08544 


\section{PACIFIC JOURNAL OF MATHEMATICS}

\section{EDITORS}

DONALD BABBITT (Managing Editor)

University of California

Los Angeles, CA 90024

Hugo RossI

University of Utah

Salt Lake City, UT 84112

C. C. MOORE and ARThur Agus

University of California

Berkeley, CA 94720
J. DugundJI

Department of Mathematics

University of Southern California

Los Angeles, CA 90007

R. FINN and J. MILGRAM

Stanford University

Stanford, CA 94305

\section{ASSOCIATE EDITORS}
R. ARENS
E. F. BeCKENBACH
B. H. NeumanN
F. WOLF
K. YoSHIDA

\section{SUPPORTING INSTITUTIONS}

UNIVERSITY OF ARIZONA

UNIVERSITY OF BRITISH COLUMBIA

CALIFORNIA INSTITUTE OF TECHNOLOGY

UNIVERSITY OF CALIFORNIA

MONTANA STATE UNIVERSITY

UNIVERSITY OF NEVADA, RENO

NEW MEXICO STATE UNIVERSITY

OREGON STATE UNIVERSITY

\author{
UNIVERSITY OF OREGON \\ UNIVERSITY OF SOUTHERN CALIFORNIA \\ STANFORD UNIVERSITY \\ UNIVERSITY OF AAWAII \\ UNIVERSITY OF TOKYO \\ UNIVERSITY OF UTAH \\ WASHINGTON STATE UNIVERSITY \\ UNIVERSITY OF WASHINGTON
}

The Supporting Institutions listed above contribute to the cost of publication of this Journal, but they are not owners or publishers and have no responsibility for its content or policies,

Mathematical parers intended for publication in the Pacific Journal of Mathematics should be in typed form or offset-reproduced, (not dittoed), double spaced with large margins. Please do not use built up fractions in the text of the manuscript. However, you may use them in the displayed equations. Underline Greek letters in red, German in green, and script in blue. The first paragraph or two must be capable of being used separately as a synopsis of the entire paper. Please propose a heading for the odd unmbered pages of less than 35 characters. Manuscripts, in triplicate, may be sent to any one of the editors. Please classify according to the scheme of Math. Reviews, Index to Vol. 39. Supply name and address of author to whom proofs should be sent. All other communications should be addressed to the managing editor, or Elaine Barth, University of California, Los Angeles, California, 90024.

50 reprints to each author are provided free for each article, only if page charges have been substantially paid. Additional copies may be obtained at cost in multiples of 50 .

The Pacific Journal of Mathematics is issued monthly as of January 1966, Regular subscription rate: $\$ 114.00$ a year (6 Vol., 12 issues). Special rate: $\$ 57.00$ a year to individual members of supporting institution.

Subscriptions, orders for numbers issued in the last three calendar years, and changes of address shoud be sent to Pacific Journal of Mathematics, P.O. Box 969, Carmel Valley, CA 93924, U.S.A. Old back numbers obtainable from Kraus Periodicals Co., Route 100, Millwood, NY 10546.

\section{PUBLISHED BY PACIFIC JOURNAL OF MATHEMATICS, A NON-PROFIT CORPORATION}

Printed at Kokusai Bunken Insatsusha (International Academic Printing Co., Ltd.). 8-8, 3-chome, Takadanobaba, Shinjuku-ku, Tokyo 160, Japan. 


\section{Pacific Journal of Mathematics}

\section{Vol. 102, No. 2 \\ February, 1982}

Richard A. Boyce, Irreducible representations of finite groups of Lie type through block theory and special conjugacy classes ...............253

Robert Jay Daverman and Dennis J. Garity, Intrinsically

$(n-2)$-dimensional cellular decompositions of $E^{n} \ldots \ldots \ldots \ldots 275$

Juan Ferrera, Spaces of weakly continuous functions ................285

William George Frederick, $\mu$-theta functions ................... 293

Christopher George Gibson and T. D. Ward, On stratifying pairs of linear

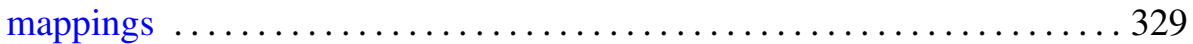

Stanley Joseph Gurak, Minimal polynomials for Gauss circulants and cyclotomic units ........................................ 347

Joachim Georg Hartung, On two-stage minimax problems ............. 355

Robert P. Kaufman, Hausdorff measure, BMO, and analytic functions . . . . 369

Neal I. Koblitz, $p$-adic analog of Heine's hypergeometric $q$-series . . . . . . . 373

Kurt Kreith, Picone-type theorems for hyperbolic partial differential

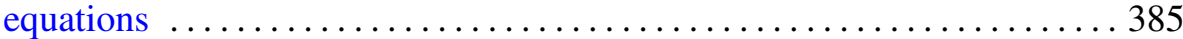

Nicholas J. Kuhn, The geometry of the James-Hopf maps ............. 397

Donald Michael Redmond, Explicit formulae for a class of Dirichlet

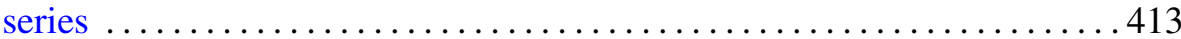

J. R. Respess and Elliott Ward Cheney, Jr., Best approximation problems in tensor-product spaces . .............................. 437

Allen Ross Schweinsberg, The operator equation $A X-X B=C$ with normal $A$ and $B$

Hans-Willi Siegberg and Guentcho Svetoslavov Skordev, Fixed point index and chain approximations

Kondagunta Sundaresan, Geometry and nonlinear analysis in Banach spaces 